\title{
Time-of-Flight Measurements of Single-Electron Wave Packets in Quantum Hall Edge States
}

\author{
M. Kataoka, ${ }^{1, *}$ N. Johnson, ${ }^{1,2}$ C. Emary, ${ }^{3, *}$ P. See, ${ }^{1}$ J. P. Griffiths, ${ }^{4}$ G. A. C. Jones,${ }^{4}$ I. Farrer, ${ }^{4, \dagger}$ \\ D. A. Ritchie, ${ }^{4}$ M. Pepper, ${ }^{2}$ and T. J. B. M. Janssen ${ }^{1}$ \\ ${ }^{1}$ National Physical Laboratory, Hampton Road, Teddington, Middlesex TW11 OLW, United Kingdom \\ ${ }^{2}$ London Centre for Nanotechnology, and Department of Electronic and Electrical Engineering, \\ University College London, Torrington Place, London WC1E 7JE, United Kingdom \\ ${ }^{3}$ Department of Physics and Mathematics, University of Hull, Kingston-upon-Hull HU6 7RX, United Kingdom \\ ${ }^{4}$ Cavendish Laboratory, University of Cambridge, J. J. Thomson Avenue, Cambridge CB3 OHE, United Kingdom
}

(Received 9 December 2015; published 22 March 2016)

\begin{abstract}
We report time-of-flight measurements on electrons traveling in quantum Hall edge states. Hot-electron wave packets are emitted one per cycle into edge states formed along a depleted sample boundary. The electron arrival time is detected by driving a detector barrier with a square wave that acts as a shutter. By adding an extra path using a deflection barrier, we measure a delay in the arrival time, from which the edgestate velocity $v$ is deduced. We find that $v$ follows $1 / B$ dependence, in good agreement with the $\vec{E} \times \vec{B}$ drift. The edge potential is estimated from the energy dependence of $v$ using a harmonic approximation.
\end{abstract}

DOI: 10.1103/PhysRevLett.116.126803

Electronic analogues of photonic quantum-optics experiments, so-called "electron quantum optics," can be performed using the beams of single-electron wave packets. The demonstration of entanglement and multiparticle interference with such wave packets would set the stage for quantum-technology applications such as quantum information processing [1]. Various theoretical proposals [2-7] and experimental realizations [8-17] employ quantum Hall edge states [18] as electron waveguides. The group velocity and dispersion relation of edge states are important parameters for understanding and controlling electron wave packet propagation. For edge magnetoplasmons, the velocity can be deduced by time-of-flight measurements with gate pulses [19-22]. Such direct velocity measurements have been difficult with electron wave packets because gate pulses would also affect the background Fermi sea. Previous experiments $[14,23,24]$ use other types of electron-transport data to estimate the electron velocity. Furthermore, electronelectron interactions can cause the formation of multiple collective modes traveling at different velocities, leading to decoherence $[14,17,25]$. In order to perform the measurements of bare group velocity by time-resolved methods, we need a robust edge-state waveguide system where the interactions between the transmitted electrons and other electrons in the background can be suppressed.

In this Letter, we demonstrate an experimental method for probing the bare edge-state velocity of electrons traveling in a depleted edge of a two-dimensional system. Electrons are emitted from a tunable-barrier single-electron pump [26-28] approximately $100 \mathrm{meV}$ above the Fermi energy [13,29]. These electrons are injected into an edge where the background two-dimensional electron gas (2DEG) is depleted to avoid the influence of electron-electron interactions. The arrival time of these wave packets is detected by an energyselective detector barrier with a picosecond resolution $[13,16]$. The travel length between the source and detector is switched by a deflection barrier. The time of flight of the extra path is measured as a delay in the arrival time at the detector [30]. The edge-state velocity is calculated from the length of the extra path and the time of flight. We find that the edge-state velocity is inversely proportional to the applied magnetic field in good agreement with the $\vec{E} \times \vec{B}$ drift velocity, where $\vec{E}$ is the electric field and $\vec{B}$ is the magnetic field. We probe the dispersion of the edge states by controlling the electron emission energy. From the energy dependence of the velocity, we deduce the edge potential profile and obtain the spatial positions of the edge states.

The measurements presented in this work are performed on two samples, $A$ and $B$, with slightly different device parameters. Figure 1(a) shows a scanning electron micrograph of a device with the same gate design as sample $B$. Both samples are made from $\mathrm{GaAs} / \mathrm{AlGaAs}$ heterostructures with a 2DEG $90 \mathrm{~nm}$ below the surface, but the 2DEG carrier density is slightly different $\left(1.8 \times 10^{15} \mathrm{~m}^{-2}\right.$ for sample $A$ and $1.6 \times 10^{15} \mathrm{~m}^{-2}$ for sample $B$ ). The active part of the device is defined by shallow chemical etching and $\mathrm{Ti} / \mathrm{Au}$ metal deposition using electron-beam lithography. The device comprises five surface gates: the pump entrance gate $(G 1)$, pump exit gate $(G 2)$, detector gate $(G 3)$, deflection gate $(G 4)$, and depletion gate $(G 5) . L_{1}$ is the path length along the deflection gate and is the same for both samples $(1.5 \mu \mathrm{m}) . L_{2}$ is the path length along the loop section defined by shallow etching, and is $2 \mu \mathrm{m}$ for sample $A$ and $5 \mu \mathrm{m}$ for sample $B$. The measurements are performed at $300 \mathrm{mK}$.

Figure 1(a) also shows the measurement circuit. The rf sine signal $V_{G 1}^{\mathrm{rf}}$ (peak-to-peak amplitude $\sim 1 \mathrm{~V}$ ) applied to 


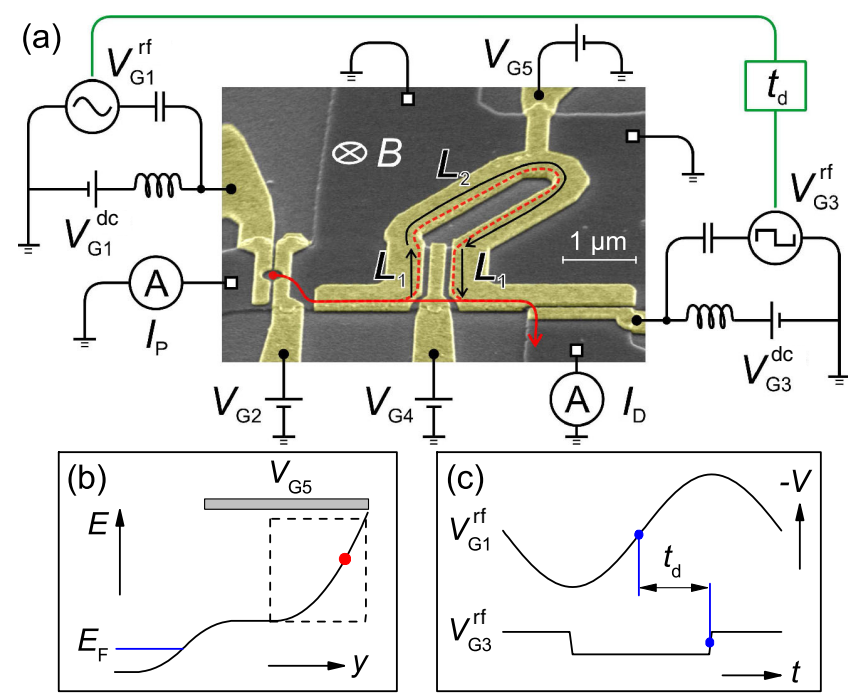

FIG. 1. (a) A scanning electron micrograph of a device and schematics of the measurement circuit. (b) Schematics of the lowest Landau level at the sample edge. Electrons travel in high energy states indicated by a red dot. The dashed box represents a region where a harmonic approximation is used to deduce the edge potential profile shown in Fig. 3(d). (c) Sine-wave signal $V_{G 1}^{\mathrm{rf}}$ applied to $G 1$ and square-wave signal $V_{G 3}^{\mathrm{rf}}$ applied to $G 3$. Their relative phase delay $t_{d}$ is controlled at a picosecond resolution using the internal skew control of the arbitrary waveform generator.

G1 pumps electrons over the barrier formed by the dc voltage $V_{G 2}$ applied on $G 2$ [27]. The rf signal is repeated periodically at a frequency $f=240 \mathrm{MHz}$, producing the pump current $I_{P}$. When the device pumps exactly one electron per cycle, $I_{P}=e f \approx 38 \mathrm{pA}$, where $e$ is the elementary charge (see the Supplemental Material for pump tuning [31]). In a magnetic field $B$ applied perpendicular to the plane of the 2DEG [in the direction indicated in Fig. 1(a)], the electrons emitted from the pump follow the sample boundary and enter the region where the background 2DEG is depleted by the negative voltage $V_{G 5}$ on $G 5$ (at least $100 \mathrm{mV}$ past the typical depletion voltage of $\sim-0.2 \mathrm{~V}$ ). The bottom of the lowest Landau level is raised above the Fermi energy $E_{F}$ but is kept lower than the electron emission energy, as shown in Fig. 1(b). The electrons travel along the edge approximately $500 \mathrm{~nm}$ (roughly equal to the extent that $G 5$ covers from the edge defined by shallow etching) away from the nearest 2DEG [as indicated by the red dot in Fig. 1(b)].

Depending on the voltage $V_{G 4}$ applied to $G 4$, the electron wave packets reach the detector $(G 3)$ through either the shorter route [solid red line in Fig. 1(a)] or the longer route (dashed line). In both cases, the majority of electrons reach the detector without measurable energy loss for the magnetic field considered here. Electrons that lose energy through LO-phonon emission $[13,35]$ are reflected by the detector barrier and do not contribute to the detector current. The longer route adds an extra length $2 L_{1}+L_{2}$ to the electron path, causing a delay in the arrival time at the detector. The arrival time is detected using a timedependent signal $[13,16]$. A square wave $V_{G 3}^{\mathrm{rf}}$ with a peakto-peak amplitude of $\sim 20 \mathrm{mV}$ is applied to $G 3$ [16] in addition to a dc voltage $V_{G 3}^{\mathrm{dc}}$. The detector current $I_{D}$ is monitored as $V_{G 3}^{\mathrm{dc}}$ is swept and the relative delay time $t_{d}$ between $V_{G 1}^{\mathrm{rf}}$ and $V_{G 3}^{\mathrm{rf}}$ [see Fig. 1(c)] is varied with a picosecond resolution [16] (see the Supplemental Material for more information [31]).

Figures 2(a)-2(c) show the behavior of the detector current for three values of $V_{G 4}$ taken at $B=14 \mathrm{~T}$ with sample $A$. Here, $d I_{D} / d V_{G 3}^{\mathrm{dc}}$ is plotted in color scale as a function of $V_{G 3}^{\mathrm{dc}}$ and $t_{d}$. The pump current is set at the quantized value for one electron emission per cycle (i.e., $I_{P} \approx e f$ ). When the detector barrier is sufficiently low (i.e., $V_{G 3}^{\text {dc }}$ is less negative) but is kept above $E_{F}$, all emitted electrons that do not suffer energy loss during the travel enter the detector contact and contribute to $I_{D}$. Therefore, $I_{D} \approx I_{P}$ as the LO-phonon emission is negligible at $B=14 \mathrm{~T}$ in these samples. When the detector barrier is sufficiently high, all electrons are blocked, and $I_{D}=0$. When the detector barrier is matched to the energy of incoming electrons, a peak in $d I_{D} / d V_{G 3}^{\mathrm{dc}}$ appears $[13,16]$. The peak position (or the detector threshold) in $V_{G 3}^{\mathrm{dc}}$ depends on $t_{d}$ because a square wave is applied to the detector gate and the sum $V_{G 3}^{\mathrm{dc}}+V_{G 3}^{\mathrm{rf}}$ determines the detector barrier height. When $t_{d}$ is small (large), electrons arrive when the square wave is negative (positive), and, hence, it shifts the detector threshold to more positive (negative) in $V_{G 3}^{\mathrm{dc}}$. The transition of the detector threshold
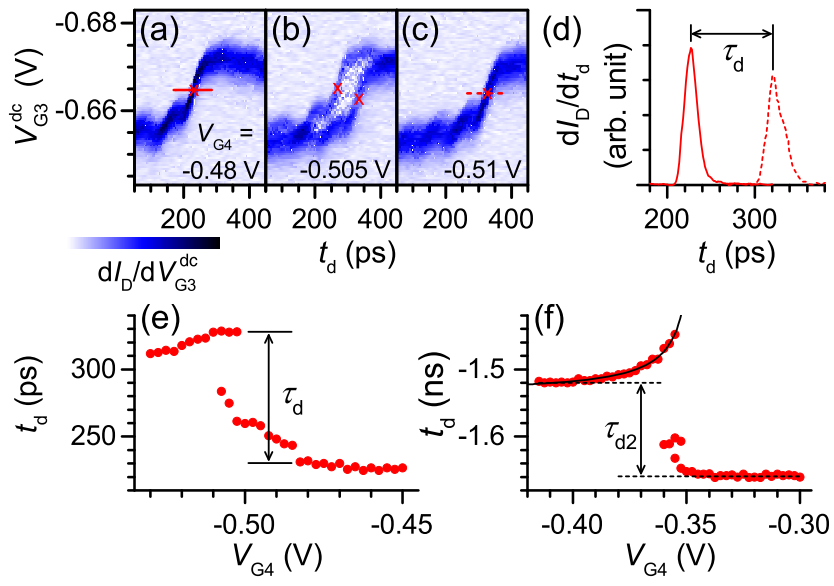

FIG. 2. (a)-(c) $d I_{D} / d V_{G 3}^{\mathrm{dc}}$ plotted in color scale as a function of $V_{G 3}^{\mathrm{dc}}$ and $t_{d}$ for three values of $V_{G 4}$. Red crosses are placed at the center of the transition in the detector threshold, which indicates the peak in the electron arrival time. The data are taken from sample $A$. (d) $d I_{D} / d t_{d}$ plotted as a function of $t_{d}$ for two values of $V_{G 4}$ : $-0.48 \mathrm{~V}$ (solid line) and $-0.51 \mathrm{~V}$ (dashed line). (e) Peak arrival time plotted as a function of $V_{G 4}$, taken from sample $A$. (f) Peak arrival time taken from sample $B$. The solid line is fit to $-1 / V_{G 4}$. 
in $V_{G 3}^{\mathrm{dc}}$ from more positive to more negative occurs at $t_{d}$, where the square-wave transition coincides with the electron arrival at the detector. As $V_{G 4}$ is made more negative, the detector transition shows splitting [Fig. 2(b)] and finally settles to larger $t_{d}$ [Fig. 2(c)]. The splitting happens as $G 4$ splits the wave packets into the shorter and longer routes, and, hence, two sets of electron wave packets arrive at the detector with a time delay. The shift of the detector transition to larger $t_{d}$ occurs because the longer route causes a delay in the arrival time.

In Fig. 2(d), $d I_{D} / d t_{d}$ is plotted as $t_{d}$ is swept through the center point of the detector transition marked by red crosses for the cases of $V_{G 4}=-0.48 \mathrm{~V}$ (solid line) in Fig. 2(a) and $-0.51 \mathrm{~V}$ (dashed line) in Fig. 2(c). These two curves represent the arrival-time distributions for the shorter and longer routes, and, hence, the time difference $\tau_{d}$ between the two peaks is the time of flight of the extra path $\left(2 L_{1}+L_{2}\right)$ taken by the longer route. The edge-state velocity in the extra path can be calculated as $v=\left(2 L_{1}+L_{2}\right) / \tau_{d}$. In this example, $v=5 \mu \mathrm{m} / 95 \mathrm{ps}=5.3 \times 10^{4} \mathrm{~m} / \mathrm{s}$.

The uncertainty in these velocity estimates arises from the uncertainties in $2 L_{1}+L_{2}$ and $\tau_{d}$. The value of $2 L_{1}+L_{2}$ is likely to be accurate only to $\pm 10 \%$, as we can only estimate it from the device geometry. This gives the same systematic error to all velocity estimates within the same sample, and, hence, it does not affect the discussions in the later sections qualitatively. The uncertainty in $\tau_{d}$ is more problematic. This is because the arrival time does not necessarily switch between just two values as the edge-state path is switched. As plotted in Fig. 2(e), the arrival time initially changes slowly towards larger $t_{d}$ as $V_{G 4}$ is made more negative. Then it starts to move through a series of small steps until it makes a final large step. After that, the arrival time moves gradually back to smaller $t_{d}$. This behavior can be interpreted as follows. The series of changes for $-0.51<V_{G 4}<-0.48 \mathrm{~V}$ occurs as the path length and the velocity of the edge state under $G 4$ is altered in a complicated manner due to disorder potential. This lasts until the edge state is finally pushed out of the region under $G 4$ at $V_{G 4} \sim-0.51 \mathrm{~V}$, and is switched to the longer route (see the Supplemental Material for more details on this effect [31]). Then the arrival time continues to change as $V_{G 4}$ is made more negative, because the velocity along $G 4$ keeps increasing as the edge potential along $G 4$ is made steeper (due to the $\vec{E} \times \vec{B}$ drift as discussed later). For the measurements with sample $A$, we take $\tau_{d}$ to be the difference in arrival time before and after rapid changes, as indicated in Fig. 2(e). A typical uncertainty in the $\tau_{d}$ estimate by this method is \pm 5 ps.

A more rigorous velocity estimate can be introduced by excluding the contribution from the electron paths along $G 4$. Figure 2(f) shows the time-of-flight data taken from sample $B$ plotted in the same manner with Fig. 2(e). With sample $B$, the arrival time changes more rapidly as $V_{G 4}$ is made more negative after the electron path is switched to the longer route. As in the case with sample $A$, this is considered to result from a rapid change in the velocity along $G 4$, and is the main source of the uncertainty in velocity estimates. In order to reduce the uncertainty, we break up the time of flight into two parts, $\tau_{d 1}$ along $G 4$ (length $2 L_{1}$ ) and $\tau_{d 2}$ along the loop (length $L_{2}$ ), i.e., $\tau_{d}=\tau_{d 1}+\tau_{d 2}=2 L_{1} / v_{1}+L_{2} / v_{2}$, where $v_{1(2)}$ is the velocity along the path $L_{1(2)}$. From this, one can see $\tau_{d} \rightarrow \tau_{d 2}=L_{2} / v_{2}$ in the limit $v_{1} / v_{2} \gg 2 L_{1} / L_{2}$. Once the electron path is deflected, $v_{1}$ increases as $V_{G 4}$ is made more negative, whereas $v_{2}$ is unaffected. Therefore, in the limit of large negative $V_{G 4}$, the time of flight settles to $\tau_{d 2}$. It is not trivial to know exactly how $v_{1}$ changes with $V_{G 4}$, but a linear relation $\left(v_{1} \propto-V_{G 4}\right)$ fits well to the experimental data [solid line in Fig. 2(f)]. As shown in Fig. 2(f), $\tau_{d 2}$ can be estimated as the difference between the saturated values of arrival time at the positive and negative ends of $V_{G 4}$. The velocity around the loop is calculated as $v_{2}=L_{2} / \tau_{d 2}$. We find that the uncertainty is reduced approximately by a factor of 3 using this method. We note that we cannot apply this method to sample $A$ as $L_{2}$ is too small to observe the saturation in the arrival time at the negative end in $V_{G 4}$.

Now, we investigate the magnetic-field and emissionenergy dependence of the velocity to see if the depleted edge-state system is consistent with an interaction-free quantum Hall edge-state model. Figure 3(a) shows the
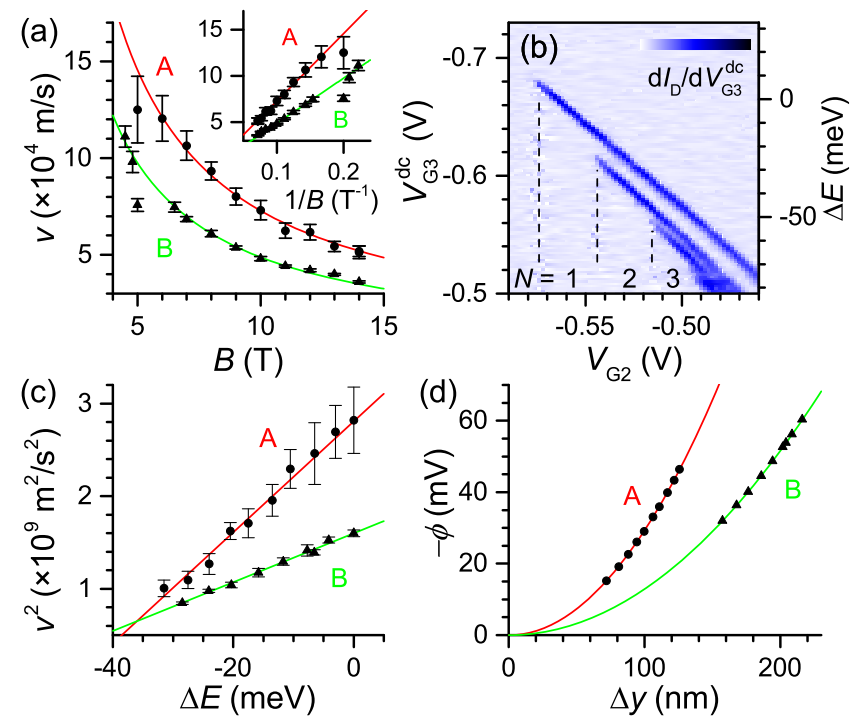

FIG. 3. (a) Edge-state velocity $v$ measured as a function of $B$. Circle (triangle) data points are taken with sample $A(B)$. The solid curves are fits to $1 / B$. Inset: $v$ plotted against $1 / B$. (b) Electron emission-energy spectrum measured at $B=14 \mathrm{~T}$. (c) $v^{2}$ plotted as a function of relative emission energy $\Delta E$. Solid lines are fits to a linear relation. (d) Edge-confinement potential $-\phi$ (solid lines) estimated from the velocity measurements. The spatial positions of the edge states corresponding to the velocity measurements are indicated by symbols. 
magnetic field dependence of the measured edge-state velocity for both samples $[v$ (the velocity along the whole extra path, $2 L_{1}+L_{2}$ ) is plotted for sample $A$ and $v_{2}$ (the velocity along the loop section $L_{2}$ only) for sample $B$ ]. Clear $1 / B$ dependence is observed for both samples down to $B=5 \mathrm{~T}$. This is in good agreement with the $\vec{E} \times \vec{B}$ drift velocity, where $v=|\vec{E} \times \vec{B}| / B^{2} \propto 1 / B$, and $\vec{E}$ is the electric field due to the edge potential.

In order to estimate the edge-confinement potential and the spatial position of edge states, we consider the dispersion relation in a quasi-one-dimensional channel with a harmonic approximation [37]. For the lowest branch of the magnetoelectric subband [38],

$$
E\left(k_{x}\right)=\epsilon_{0}+\frac{1}{2} \hbar \Omega+\frac{\hbar^{2} k_{x}^{2}}{2 m^{*}} \frac{\omega_{y}^{2}}{\omega_{y}^{2}+\omega_{c}^{2}},
$$

where $k_{x}$ is the wave number in the edge-state transport direction (in the $x$ direction), $\epsilon_{0}$ is the lowest twodimensional subband energy, $\hbar \omega_{y}$ is the transverse confinement energy (in the $y$ direction), $\hbar \omega_{c}$ is the cyclotron energy, and $m^{*}$ is the electron effective mass. From the dispersion relation and the group velocity $v=(1 / \hbar)(d E / d k)$, one can deduce

$$
\frac{1}{2} m^{*} v^{2} \approx \frac{\omega_{y}^{2}}{\omega_{c}^{2}}\left(E-\epsilon_{0}-\frac{1}{2} \hbar \omega_{c}\right),
$$

in the limit of large magnetic field $\left(\omega_{c} \gg \omega_{y}\right)$. From this, $\omega_{y}$ can be deduced by plotting $v^{2}$ against $E$.

The emission energy from our single-electron source can be tuned over a wide range [13]. This can be used to probe the energy dependence of the edge-state velocity. Figure 3(b) shows the emission energy spectrum measured as $V_{G 2}$ is varied and with a static detector barrier $\left(V_{G 3}^{\mathrm{rf}}=0\right)$ with electrons traveling along the longer route $\left(V_{G 4}=-0.7 \mathrm{~V}\right)$ at $B=14 \mathrm{~T}$. The conversion to relative emission energy $\Delta E$ [shown on the right-hand vertical axis in Fig. 3(b) with the highest energy point used in this work set as zero] is made by calibrating $V_{G 3}^{\text {dc }}$ against LO-phonon emission peaks [13] (not visible in this particular data set), assuming the LO-phonon energy of $36 \mathrm{meV}$ [39] (see the Supplemental Material for more details on this procedure [31]). The electron emission energy decreases linearly as $V_{G 2}$ is made more positive.

Figure 3(c) plots $v^{2}$ measured as a function of relative emission energy $\Delta E$ at $B=14 \mathrm{~T}$ for both samples. As expected, they fit well to straight lines. From these, we deduce $\hbar \omega_{y}=2.7$ and $1.8 \mathrm{meV}$, and the bottom of the confinement potential at $\Delta E=-47$ and $-61 \mathrm{meV}$, for samples $A$ and $B$, respectively. We can then reconstruct the edge-confinement potential $\phi=-m^{*} \omega_{y}^{2} y^{2} / 2 e$ as shown in Fig. 3(d). Here, we set the potential at the bottom of the parabola as zero. From each data point in Fig. 3(c), we can deduce the potential energy $-e \phi$ at the position of the guiding center by subtracting the kinetic energy $\frac{1}{2} m^{*} v^{2}$ from the total (relative) energy $\Delta E$. We can then visualize the spatial position of the edge states as plotted in Fig. 3(d).

In summary, we have shown the measurements of the time-of-flight of electron wave packets traveling through edge states. The electrons travel in the region where the background 2DEG is depleted and electron-electron interaction is minimized. We find that the electron velocity is in good agreement with the expected $\vec{E} \times \vec{B}$ drift. From the energy dependence, we deduce the edge-confinement potential. Our technique provides a way of characterizing the edge-state transport of single-electron wave packets with picosecond resolutions. The method that we have developed to transport electron wave packets in depleted edges could provide an ideal electron waveguide system where decoherence due to interactions can be avoided for electron quantum-optics experiments.

We would like to thank Heung-Sun Sim and Sungguen Ryu for useful discussions. This research was supported by the UK Department for Business, Innovation and Skills, NPL's Strategic Research Programme, and the UK EPSRC.

*masaya.kataoka@npl.co.uk

†Present address: Department of Electronic and Electrical Engineering,

University of Sheffield, Mappin Street, Sheffield S1 3JD, United Kingdom.

*Present address: Joint Quantum Centre Durham-Newcastle, School of Mathematics and Statistics, Newcastle University, Newcastle upon Tyne NE1 7RU, United Kingdom.

[1] C. H. Bennett and D. P. DiVincenzo, Nature (London) 404, 247 (2000).

[2] P. Samuelsson, E. V. Sukhorukov, and M. Büttiker, Phys. Rev. Lett. 92, 026805 (2004).

[3] S. Ol'khovskaya, J. Splettstoesser, M. Moskalets, and M. Büttiker, Phys. Rev. Lett. 101, 166802 (2008).

[4] J. Splettstoesser, M. Moskalets, and M. Büttiker, Phys. Rev. Lett. 103, 076804 (2009).

[5] M. Moskalets and M. Büttiker, Phys. Rev. B 83, 035316 (2011).

[6] G. Haack, M. Moskalets, J. Splettstoesser, and M. Büttiker, Phys. Rev. B 84, 081303(R) (2011).

[7] F. Battista and P. Samuelsson, Phys. Rev. B 85, 075428 (2012).

[8] Y. Ji, Y. Chung, D. Sprinzak, M. Heiblum, D. Mahalu, and H. Shtrikman, Nature (London) 422, 415 (2003).

[9] M. Henny, S. Oberholzer, C. Strunk, T. Heinzel, K. Ensslin, M. Holland, and C. Schönenberger, Science 284, 296 (2008).

[10] G. Fève, A. Mahé, J.-M. Berroir, T. Kontos, B. Plaçais, D. C. Glattli, A. Cavanna, B. Etienne, and Y. Jin, Science 316, 1169 (2007).

[11] E. Bocquillon, F. D. Parmentier, C. Grenier, J.-M. Berroir, P. Degiovanni, D. C. Glattli, B. Plaçais, A. Cavanna, Y. Jin, and G. Fève, Phys. Rev. Lett. 108, 196803 (2012). 
[12] E. Bocquillon, V. Freulon, J.-M. Berroir, P. Degiovanni, B. Plaçais, A. Cavanna, Y. Jin, and G. Fève, Science 339, 1054 (2013).

[13] J. D. Fletcher, P. See, H. Howe, M. Pepper, S. P. Giblin, J. P. Griffiths, G. A. C. Jones, I. Farrer, D. A. Ritchie, T. J. B. M. Janssen, and M. Kataoka, Phys. Rev. Lett. 111, 216807 (2013).

[14] E. Bocquillon, V. Freulon, J.-M. Berroir, P. Degiovanni, B. Plaçais, A. Cavanna, Y. Jin, and G. Fève, Nat. Commun. 4, 1839 (2013).

[15] N. Ubbelohde, F. Hohls, V. Kashcheyevs, T. Wagner, L. Fricke, B. Kästner, K. Pierz, H. W. Schumacher, and R. J. Haug, Nat. Nanotechnol. 10, 46 (2015).

[16] J. Waldie, P. See, V. Kashcheyevs, J. P. Griffiths, I. Farrer, G. A. C. Jones, D. A. Ritchie, T. J. B. M. Janssen, and M. Kataoka, Phys. Rev. B 92, 125305 (2015).

[17] V. Freulon, A. Marguerite, J.-M. Berroir, B. Plaçais, A. Cavanna, Y. Jin, and G. Fève, Nat. Commun. 6, 6854 (2015).

[18] B. I. Halperin, Phys. Rev. B 25, 2185 (1982).

[19] R. C. Ashoori, H. L. Stormer, L. N. Pfeiffer, K. W. Baldwin, and K. West, Phys. Rev. B 45, 3894 (1992).

[20] N. B. Zhitenev, R. J. Haug, K. v. Klitzing, and K. Eberl, Phys. Rev. B 49, 7809 (1994).

[21] H. Kamata, T. Ota, K. Muraki, and T. Fujisawa, Phys. Rev. B 81, 085329 (2010).

[22] N. Kumada, H. Kamata, and T. Fujisawa, Phys. Rev. B 84, 045314 (2011).

[23] D. T. McClure, Y. Zhang, B. Rosenow, E. M. LevensonFalk, C. M. Marcus, L. N. Pfeiffer, and K. W. West, Phys. Rev. Lett. 103, 206806 (2009).

[24] F. Martins, S. Faniel, B. Rosenow, M. G. Pala, H. Sellier, S. Huant, L. Desplanque, X. Wallart, V. Bayot, and B. Hackens, New J. Phys. 15, 013049 (2013).

[25] D. Ferraro, B. Roussel, C. Cabart, E. Thibierge, G. Fève, Ch. Grenier, and P. Degiovanni, Phys. Rev. Lett. 113, 166403 (2014).

[26] M. D. Blumenthal, B. Kaestner, L. Li, S. Giblin, T. J. B. M. Janssen, M. Pepper, D. Anderson, G. Jones, and D. A. Ritchie, Nat. Phys. 3, 343 (2007).

[27] B. Kaestner, V. Kashcheyevs, S. Amakawa, M. D. Blumenthal, L. Li, T. J. B. M. Janssen, G. Hein, K. Pierz, T. Weimann, U. Siegner, and H. W. Schumacher, Phys. Rev. B 77, 153301 (2008).
[28] B. Kaestner, V. Kashcheyevs, G. Hein, K. Pierz, U. Siegner, and H. W. Schumacher, Appl. Phys. Lett. 92, 192106 (2008).

[29] C. Leicht, P. Mirovsky, B. Kaestner, F. Hohls, V. Kashcheyevs, E. V. Kurganova, U. Zeitler, T. Weimann, K. Pierz, and H. W. Schumacher, Semicond. Sci. Technol. 26, 055010 (2011).

[30] One may argue that time-of-flight measurements could be performed by using two detector gates on the electron path, and detecting the difference in the arrival time between the two detectors. However, in this method, synchronizing two detector signals with a picosecond resolution would be challenging.

[31] See Supplemental Material at http://link.aps.org/ supplemental/10.1103/PhysRevLett.116.126803, which includes Refs. [32-34], for further experimental details.

[32] M. Kataoka, J. D. Fletcher, P. See, S. P. Giblin, T. J. B. M. Janssen, J. P. Griffiths, G. A. C. Jones, I. Farrer, and D. A. Ritchie, Phys. Rev. Lett. 106, 126801 (2011).

[33] S. P. Giblin, M. Kataoka, J. D. Fletcher, P. See, T. J. B. M. Janssen, J. P. Griffiths, G. A. C. Jones, I. Farrer, and D. A. Ritchie, Nat. Commun. 3, 930 (2012).

[34] J. D. Fletcher, M. Kataoka, S. P. Giblin, S. Park, H.-S. Sim, P. See, D. A. Ritchie, J. P. Griffiths, G. A. C. Jones, H. E. Beere, and T. J. B. M. Janssen, Phys. Rev. B 86, 155311 (2012).

[35] The depletion gate can be used to suppress the energy relaxation due to LO-phonon emission [13]. With a negative voltage on $G 5$ enough to deplete the 2DEG underneath, LO-phonon scattering becomes small above $B \sim 5 \mathrm{~T}$. The suppression of LO-phonon emission can be explained as a result of decreased wave function overlap due to the shape of the confining potential [36].

[36] C. Emary, A. Dyson, S. Ryu, H.-S. Sim, and M. Kataoka, Phys. Rev. B 93, 035436 (2016).

[37] Although, in this approximation, the channel is confined by a harmonic potential whereas in our device the confinement potential is only on one side (the other edge is far away), it is still valid to use this approximation in high $B$ limit as the wave function becomes localized on one edge only.

[38] S. Datta, Electronic Transport in Mesoscopic Systems (Cambridge University Press, Cambridge, England, 1995).

[39] M. Heiblum, M. I. Nathan, D. C. Thomas, and C. M. Knoedler, Phys. Rev. Lett. 55, 2200 (1985). 